\title{
Acknowledgment to reviewers
}

\section{Spector ${ }^{1}$}

(c) Springer Science+Business Media, LLC, part of Springer Nature 2018

Genetic Programming and Evolvable Machines relies on generous contributions of time and expertise by scholars who agree to review articles on a voluntary basis. Over the past year the following people have made such contributions:

Brad Alexander
Shaukat Ali
Andrea Arcuri
Ignacio Arnaldo
R. Muhammad Atif Azad
Dylan Banarse
Amit Benbassat
Peter Bentley
Michal Bidlo
Stefano Cagnoni
Jeffrey Chan
Francis Chicano
Vic Ciesielski
German Creamer
Márjory Da Costa-Abreu
Roland Dobai
Alan Dorin
Liang Gao
Oscar Garnica
Mario Giacobini

L. Spector 1spector@hampshire.edu

$\begin{array}{ll}\text { Juan Herrero } & \text { Justyna Petke } \\ \text { Malcolm Heywood } & \text { Stjepan Picek } \\ \text { Cezary Z. Janikow } & \text { Nelishia Pillay } \\ \text { Colin Johnson } & \text { John Robinson } \\ \text { Anna Jordanous } & \text { Patricia Ryser-Welch } \\ \text { Paul Kaufmann } & \text { Yago Saez } \\ \text { Edward Keedwell } & \text { Ivan Sekaj } \\ \text { Krzysztof Krawiec } & \text { Kisung Seo } \\ \text { Pavel Kromer } & \text { Sara Silva } \\ \text { Stuart Lacy } & \text { Kevin Sim } \\ \text { William Langdon } & \text { James Smith } \\ \text { Xianneng Li } & \text { Andy Song } \\ \text { Luca Manzoni } & \text { Giovanni Squillero } \\ \text { James McDermott } & \text { Kenneth Stanley } \\ \text { Yi Mei } & \text { Thomas Stutzle } \\ \text { Maizura Mokhtar } & \text { Petr Svenda } \\ \text { Kourosh Neshatian } & \text { Jerry Swan } \\ \text { Su Nguyen } & \text { Marcin Szubert } \\ \text { Trung Thanh Nguyen } & \text { Gianluca Tempesti } \\ \text { Randy Olson } & \text { Hsing-Chih Tsai }\end{array}$

Juan Herrero

Malcolm Heywood

Colin Johnson

Anna Jordanous

Krzysztof Krawiec

William Langdon

Xianneng Li

James McDermott

Maizura Mokhtar

Kourosh Neshatian

Trung Thanh Nguyen

\author{
Nelishia Pillay \\ Patricia Ryser-Welch \\ Ivan Sekaj \\ Kisung Seo \\ James Smith \\ Kenneth Stanley \\ Petr Svenda \\ Marcin Szubert \\ Hsing-Chih Tsai
}

1 School of Cognitive Science, Hampshire College, Amherst, MA 01002, USA 


$\begin{array}{lll}\begin{array}{l}\text { Mario Gongora } \\ \text { Crina Grosan }\end{array} & \text { Michael O’Neill } & \text { Zdenek Vasicek } \\ \text { Steven Gustafson } & \text { Denis Pallez } & \text { John R. Woodward } \\ \text { DeQiang Han } & \text { Gisele Pappa } & \text { Shin Yoo } \\ & \text { Mario Pavone } & \text { Landaez Ysmaldo } \\ \text { Simon Harding } & & \text { Jose } \\ \text { Erik Hemberg } & \text { Tomasz Pawlak } & \text { Mengjie Zhang } \\ & \text { Francisco Pereira } & \text { Huiru Zheng }\end{array}$

I thank these reviewers for their service to the Genetic Programming and Evolvable Machines journal and to the research community.

Lee Spector

Editor-in-Chief, Genetic Programming and Evolvable Machines 